\title{
Optimal Check Size and Reversal Rate to Elicit Pattern-reversal MEG Responses
}

\author{
Wei-Ta Chen, Yu-Chieh Ko, Kwong-Kum Liao, Jen-Chuen Hsieh, \\ Tzu-Chen Yeh, Zin-An Wu, Low-Tone Ho, Yung-Yang Lin
}

\begin{abstract}
Objective: To determine the impact of check size and interstimulus interval (ISI) on neuromagnetic visual cortical responses. Methods: We recorded visual evoked fields to pattern-reversal stimulation with central occlusion in ten subjects. The $\sim 100 \mathrm{~ms}$ magnetic activation (P100m) was analyzed by single dipole modeling. Results: With 1 s ISI, P100m strengths increased as check size increased from 15' up to 120' of visual arc, and larger checks elicited less P100m activation. With 120' checks, we found no P100m attenuation as ISI decreased from $4 \mathrm{~s}$ to $0.16 \mathrm{~s}$. P100m sources around the calcarine sulcus did not vary with check size or ISI. Conclusions: The magnitude of cortical activation during visual contrast processing is check size-dependent and the 120' checks are optimum for future studies on neuromagnetic visual cortical functions using central-occluded stimulation. The corresponding neuronal activation demonstrated a short refractory period less than $0.16 \mathrm{~s}$. We also found significantly overlapping cortical representation areas for different check sizes or ISIs.
\end{abstract}

RÉSUMÉ: Taille optimale des carreaux et taux d'inversion des réponses MEG à la stimulation du champ périphérique par damier. Objectif: Déterminer l'impact de la taille des carreaux et de l'intervalle inter stimulus (IIS) sur les réponses corticales visuelles neuromagnétiques. Méthodes: Nous avons enregistré des potentiels évoqués visuels à la stimulation par damier avec occlusion centrale chez 10 sujets. L'activation magnétique $\sim 100$ ms (P100m) a été analysée par modélisation dipôle unique. Résultats: Avec $1 \mathrm{~s} \mathrm{d'IIS,} \mathrm{les} \mathrm{forces} \mathrm{P100m} \mathrm{augmentaient}$ avec l'augmentation de la taille des carreaux de 15' jusqu'à 120' d'arc visuel. Les carreaux de plus grande taille déclenchaient moins d'activation P100m. Avec des carreaux de 120', nous n'avons observé aucune atténuation P100m avec la diminution d'IIS de $4 \mathrm{~s}$ à $0,16 \mathrm{~s}$. Les sources P100m autour de la scissure calcarine ne variaient pas selon la taille des carreaux ou l'IIS. Conclusions: L'ampleur de l'activation corticale pendant le traitement du contraste visuel est dépendant de la taille des carreaux et les carreaux de 120' sont optimaux pour l'étude des fonctions corticales visuelles neuromagnétiques utilisant l'occlusion centrale. L'activation neuronale correspondante a une période réfractaire courte de moins de $0,16 \mathrm{~s}$. Nous avons également constaté un chevauchement significatif des zones de représentation corticale pour des carreaux de tailles différentes ou des IISs différents.

Can. J. Neurol. Sci. 2005; 32: 218-224

The evoked cortical responses to pattern-reversal stimuli reflect functional processing of visual sensory inputs. ${ }^{1}$ In demyelination diseases, visual pathways may be affected in an uneven manner, ${ }^{2}$ with selective involvement in the spatial or temporal processing of visual information. ${ }^{2,3}$ Using various check sizes in recording visual evoked potentials (VEPs) may improve the detection of visual pathway dysfunctions, even in subclinical cases. ${ }^{3}$ These phenomena could be partially explained by the parallel visual processing of magnocellular and parvocellular retinal ganglion cells and their spatiotemporal reactivities. ${ }^{4}$ VEP studies in migraine ${ }^{5}$ and Alzheimer's disease ${ }^{6}$ also demonstrated visual processing deficits in specific spatiotemporal frequencies. Thus, a systematic investigation of the influence of checkerboard parameters on visual cortical responses is of neurophysiological and clinical importance.

The deflection peaking at $\sim 100 \mathrm{~ms}$ (P100) is the most consistent VEP activity. Changes in P100 responses may reflect the integrative visual dysfunction. ${ }^{3}$ Pattern stimulation with checks of $\sim 30$ minutes of arc (30') may elicit the minimal P100 latency, whereas the clearly smaller or larger checks can elicit responses with longer latencies. ${ }^{3,7}$ However, the check size effect

From the Neurological Institute (W-TC, K-KL, Z-AW, Y-YL), Department of Ophthalmology (Y-CK), and Department of Medical Research and Education (J-CH, TCY,L-TH, Y-YL), Taipei Veterans General Hospital; Department of Neurology, Taipei Medical University Hospital (W-TC); Institute of Clinical Medicine (Y-CK), School of Medicine (W-TC,Y-CK, K-KL, J-CH,T-CY,Z-AW, L-TH, Y-YL), and Institute of

Neuroscience, School of Life Science (W-TC,J-CH, Y-YL) National Yang-Ming University, Taipei, Taiwan

RECEIVED JUly 15, 2004. ACCEPTED IN FINAL FORM JANUARY 7, 2005.

Reprint requests to: Yung-Yang Lin, Neurology, Neurological Institute, Taipei Veterans General Hospital, No.201, Sec.2, Shih-Pai Rd., Taipei 112, Taiwan 
upon P100 amplitudes varied between different VEP recordings, partly because of varying methods. ${ }^{3,7}$ Comparing P100 responses between different studies would not be feasible, if the impacts of stimulus parameters (especially check size and interstimulus interval (ISI)) remained undetermined.

Magnetoencephalography (MEG) measures the synchronized neuronal activation with excellent temporal and reasonable spatial resolution. ${ }^{8}$ The source localization of visual evoked magnetic fields (VEFs) does not yield the paradoxical lateralization reported previously in VEP recordings. ${ }^{9}$ In response to pattern-reversal stimuli, the triphasic wave complex in VEFs is similar to that in VEP recordings. ${ }^{10}$ The magnetic $\sim 100 \mathrm{~ms}$ response $(\mathrm{P} 100 \mathrm{~m})$ is the most consistent activity recorded by total or peripheral field stimulation. ${ }^{11}$ Due to the radial orientation for occipital pole neurons, the responses to fovea stimulation can be better recorded by VEP than VEF recordings. In contrast, the peripheral half-field stimulation elicits tangentially oriented activation which is thus favorably detected by MEG. ${ }^{11}$

In the present study, using a whole-head MEG, we aimed to explore the effect of check size and ISI on P100m responses to central-occluded checkerboard stimulation. It was hoped that our results would help to find the optimal experimental conditions for eliciting VEFs by peripheral field stimulation.

\section{MeTHODS}

Ten healthy right-handed volunteers (six women and four men; age 20-37 years) gave their informed consent and participated in the study. They had either normal or corrected-tonormal visual acuity. This research adhered to the tenets of the Declaration of Helsinki, and the experimental protocol had a prior acceptance by the institutional review board of Taipei Veterans General Hospital.

Pattern reversal white and black checkerboard stimuli were generated using a personal computer (Acer Veriton). We delivered the visual stimuli using Presentation 0.52 NBS (Neurobehavioral Systems, Inc., CA, U.S.A.) and projected them onto a screen $\sim 110$ centimeters in front of the subject by an liquid crystal display (LCD) projector (Electrohome Electronics, 38DMD001-EXP, Canada). The mean luminance of the screen checkerboard pattern was $20 \mathrm{~cd} / \mathrm{m}^{2}$, while the contrast was maintained at 0.96 . The time lag between trigger onset and stimulus presentation on the screen was $35.0 \pm 0.6 \mathrm{~ms}$ (mean \pm standard error of the mean); this delay was subtracted from the latencies of VEFs.

We asked the subject to gaze at a tiny red fixation point in the center of the screen and then covered the right eye with an eye bandage during the recording. The total field size of the left halffield was $15^{\circ} \times 22^{\circ}$ (width $\mathrm{x}$ height, degrees of arc) in visual angle with a central occlusion of $2^{\circ}$.

This study consisted of two separate experiments. In Experiment 1, we recorded VEFs to checkerboard patterns of varying check sizes $\left(15^{\prime}, 30^{\prime}, 45^{\prime}, 60^{\prime}, 90^{\prime}, 120^{\prime}, 180^{\prime}\right.$ and 450') reversed at a rate of $1 \mathrm{~s}($ ISI $=1 \mathrm{~s})$. The size of checks eliciting maximal mean amplitude of $\mathrm{P} 100 \mathrm{~m}$ responses across subjects was defined as the optimal check size. In Experiment 2, we recorded VEFs to checkerboard stimuli of the optimum check size at various ISIs consisting of $0.16,0.18,0.25,0.5,1,2$, and 4 s. For each subject the order of stimulus presentation was randomized with respect to check size or ISI.

For VEF recordings, the subject sat comfortably in a magnetically shielded room with the head supported against the helmet-shaped bottom of a whole-scalp 306-channel neuromagnetometer (Vectorview ${ }^{\mathrm{TM}}$, Elekta Neuromag, Helsinki, Finland). The neuromagnetometer comprised 102 identical triple sensor elements, which consisted of two orthogonal planar gradiometers and one magnetometer coupled to three superconducting quantum interference devices. In this study, data analysis was based on signals of the 204 planar gradiometers.

The signals were bandpass filtered $(0.1-130 \mathrm{~Hz})$ and digitized at $500 \mathrm{~Hz}$. One hundred responses were averaged for each stimulus condition, and each trial was repeated to ensure reproducibility. Epochs coinciding with signals exceeding 300 $\mu \mathrm{V}$ in the simultaneously recorded vertical electro-oculogram were automatically rejected from the analysis.

To identify the sources of the measured signals, we visually searched those channels with signal deflections clearly exceeding the prestimulus background level and selected the time windows of cortical responses for further analysis. The single equivalent current dipole (ECD) best describing the measured data was found by a least-squares search using the subsets of 32-36 channels around the maximal responses. Goodness-of-fit of the model was calculated and only ECDs explaining more than $80 \%$ of the field variance at selected periods of time over a subset of channels were used for further analysis.

The exact location of the head with respect to MEG sensors was found by measuring magnetic signals produced by currents led to four head indicator coils at known sites on the scalp. The coil locations with respect to anatomical landmarks on the head were determined with three-dimensional digitizer to allow further alignment of MEG and magnetic resonance imaging (MRI) coordinate systems. MRIs of the subject's brain were acquired with a 3-T Bruker Medspec300 scanner or $1.5 \mathrm{~T}$ Siemens Magnetom Sonata system (Germany). Based on the spherical head model, the three-dimensional location and orientations of the ECDs calculated from source analysis were coregistered to MRIs of the subject's own brain. The positive x, $y$-, and z-axes in our head-coordinate system go towards the right preauricular point, the nasion, and the head vertex, respectively.

The peak latencies, amplitudes, and locations of $\mathrm{P} 100 \mathrm{~m}$ sources were computed as the mean of the two repeated recordings. The effects of check size or ISI on ECD latencies, amplitudes, and locations were first assessed using an analysis of variance for repeated measures. Individual differences between means were further evaluated with Bonferroni multiplecomparison procedures. $\mathrm{P}$ value below 0.05 was considered significant.

\section{Results}

Figure 1 shows the clearest neuromagnetic signal selected from the scalp sensors in response to left hemifield checkerboard 


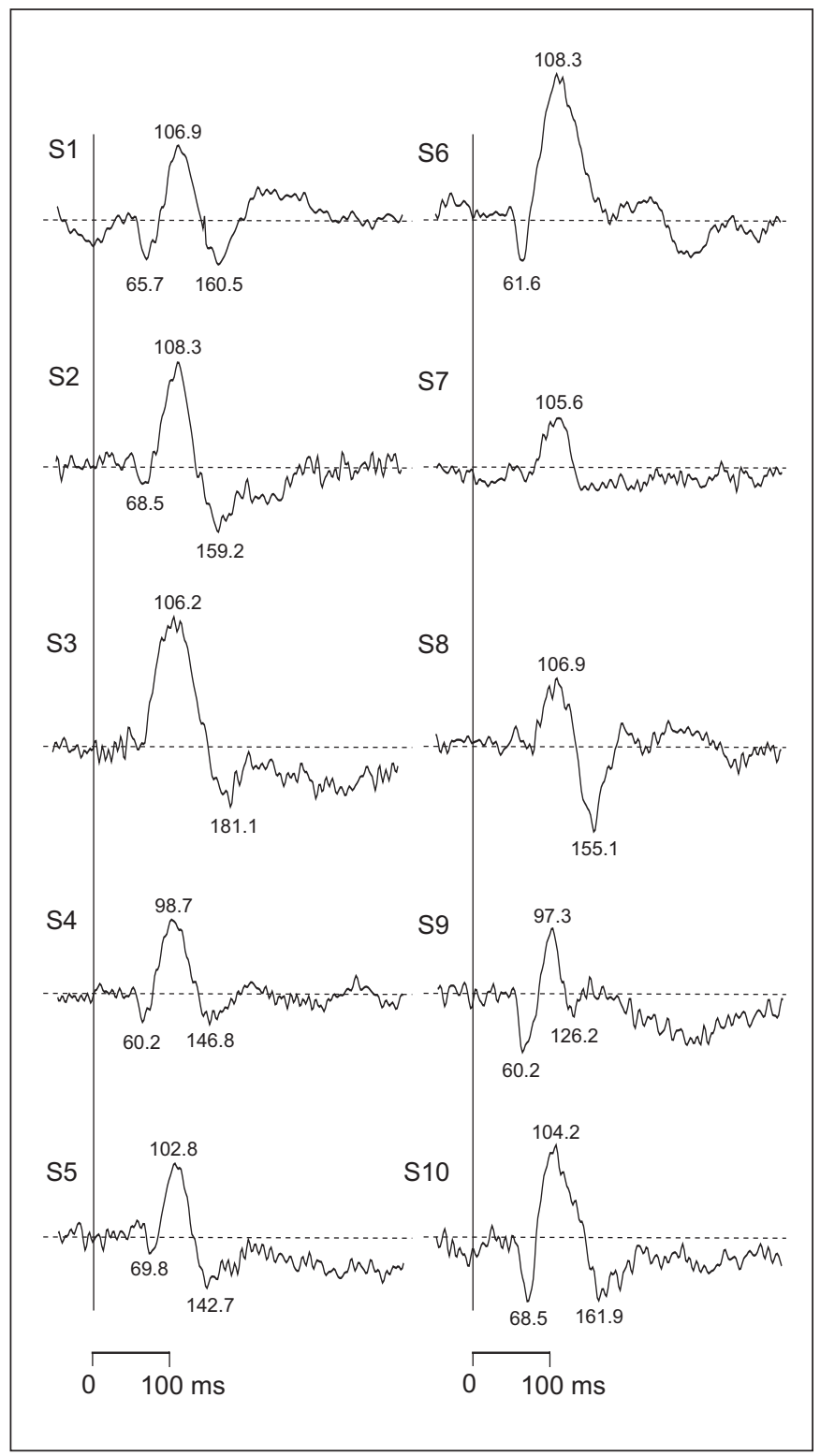

Figure 1: The neuromagnetic signals recorded from the right occipital cortex of each subject in response to left hemifield checkerboard reversals $\left(\right.$ check size $\left.=120^{\prime}\right)$. One clearest signal is selected from each subject. The signal with strongest deflection $\sim 100 \mathrm{~ms}$ is selected from all subjects. Note most signals are triphasic wave complexes. The second deflection is the most robust, consistently peaking at $\sim 100 \mathrm{~ms}$ across all subjects; the first ( 60-70 ms) and the third ( 126-182 ms) deflections are relatively less clearly identified.

reversals. Most of these signals were triphasic wave complexes located in the right occipital region. The second wave component was the most robust, peaking consistently at $\sim 100 \mathrm{~ms}(\mathrm{P} 100 \mathrm{~m})$. The first and the third deflection, nevertheless, could be clearly seen in only seven and eight subjects, respectively. The peak latencies across subjects were about $60-70 \mathrm{~ms}$ for the first deflection but more varied (126-181 ms) for the third one. Therefore, only the second wave component (P100m) was included for further analysis.

Figure 2 illustrates the topographic distribution of cortical

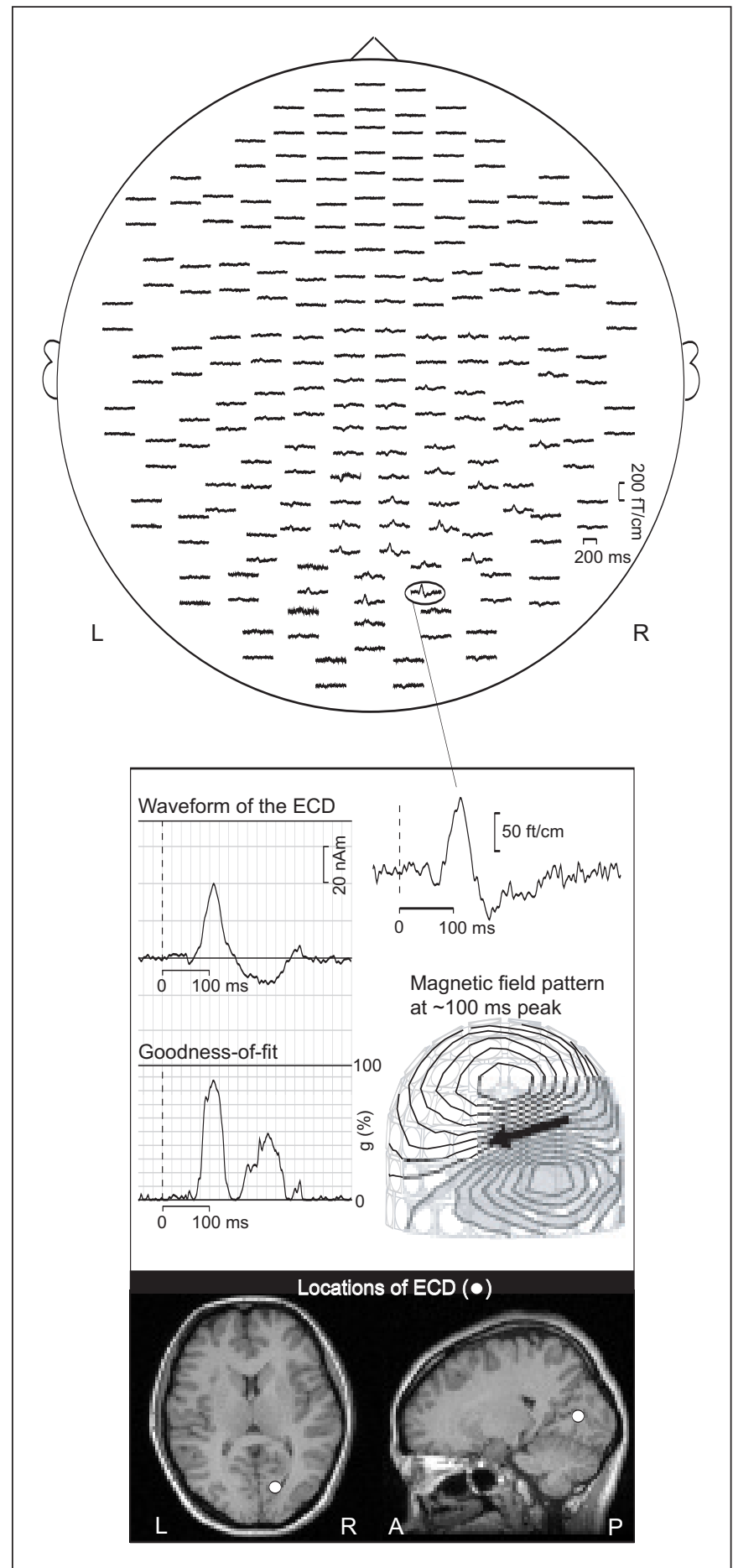

Figure 2: The spatial distribution of visual evoked fields in Subject 2 to left peripheral (2-15 degrees) checkerboard stimulation (check size = $120^{\prime}, I S I=1 \mathrm{~s}$ ). The head is viewed from the top, with nose pointing upwards. Each response doublet represents signals recorded by the two orthogonal gradiometers. The insert shows an enlarged signal (upper right) from the right occipital area, the magnetic field pattern on the helmet-shaped sensor array viewed from the back (middle right), and the MRI-overlaid locations (white dots) of the equivalent current dipole (ECD) at $100 \mathrm{~ms}$. The isocontour map has a separation of 50 femtotesla between two neighboring isocontour lines. The shaded areas indicate magnetic flux emerging from the head, and the arrow indicates the orientation of the ECD. 
Table 1: Mean amplitudes (nAm), peak latencies (ms), and locations (x-, y-, z- coordinates; mm) of P100m sources to checkerboard stimulation in ten subjects with respect to various checks

\begin{tabular}{|c|c|c|c|c|c|c|c|c|}
\hline Check size (min of arc) & 15 & 30 & 45 & 60 & 90 & 120 & 180 & 450 \\
\hline Amplitude & 21.4 & 25.5 & 28.1 & 31.6 & $36.9^{*}$ & $38.9^{*}$ & 36.7 & 28.5 \\
\hline $\mathrm{SD}$ & 11.6 & 8.3 & 9.8 & 13.7 & 11.5 & 11.2 & 12.4 & 9.0 \\
\hline Latency & 125.8 & $115.0^{*}$ & $109.7 *$ & $107.2 *$ & $104.7 *$ & $103.7 *$ & $106.1 *$ & $111.2 *$ \\
\hline $\mathrm{SD}$ & 11.9 & 10.8 & 4.3 & 4.7 & 3.5 & 4.9 & 8.0 & 10.0 \\
\hline $\mathrm{x}$ value & 20.1 & 17.7 & 14.5 & 14.1 & 15.5 & 16.2 & 15.1 & 14.8 \\
\hline SD & 13.9 & 11.5 & 7.3 & 5.1 & 5.0 & 4.7 & 7.8 & 9.5 \\
\hline y value & -35.7 & -40.1 & -40.3 & -42.4 & -40.1 & -38.4 & -36.5 & -34.8 \\
\hline SD & 10.3 & 8.0 & 8.4 & 7.3 & 9.2 & 7.8 & 5.9 & 8.7 \\
\hline $\mathrm{z}$ value & 44.9 & 45.6 & 44.0 & 44.8 & 44.0 & 44.1 & 46.8 & 51.3 \\
\hline SD & 5.0 & 4.4 & 4.7 & 4.5 & 4.0 & 4.2 & 7.4 & 10.0 \\
\hline
\end{tabular}

$* \mathrm{p}<0.01$ compared with results elicited by checks of 15 minutes of arc.

responses in Subject 2 to checkerboard stimuli presented to the left central-occluded half-field. Clear deflections were identified in the right posterior head region with peak latency at $\sim 100 \mathrm{~ms}$. With the spherical head assumption, the measured responses could be well explained by one-ECD modeling. Superimposed on the subject's own MRIs, the ECD was localized around the right calcarine fissure.

Table 1 shows the ECD amplitudes, latencies, and locations of $\mathrm{P} 100 \mathrm{~m}$ responses to the checkerboard stimuli at $1 \mathrm{~s}$ ISI with respect to various checks ranging from $15^{\prime}$ to 450 '. $\mathrm{P} 100 \mathrm{~m}$ responses to $120^{\prime}$ checks showed the largest magnitudes and shortest latencies, whereas smaller or larger checks elicited responses with reduced strengths as well as prolonged latencies. Responses to $15^{\prime}$ checks showed significantly longer latencies and smaller strengths than those elicited by larger checks (15' vs. 30 ' 450'; $p=0.005 \sim 0.007)$. The largest mean latency difference between responses to $15^{\prime}$ and $120^{\prime}$ checks was $22 \mathrm{~ms}$. In addition, the ECD locations did not change with check size.

Table 2 shows the ECD amplitudes, latencies, and locations of P100m responses to 120' checkerboard stimuli with respect to various ISIs ranging from $0.16 \mathrm{~s}$ to $4 \mathrm{~s}$. The mean strengths elicited by short ISIs ( 0.16 or $0.18 \mathrm{~s}$ ) seemed slightly larger than those by longer ISIs, but the difference was not significant. The mean latencies varied only slightly and nonsystematically with ISI changes. The maximal difference in dipole latencies was $5 \mathrm{~ms}$ (1 s vs. 4 s ISIs), clearly smaller than that ( $22 \mathrm{~ms}$ ) contributed to check size variation (15' vs. 120'). On top of that, the ECD locations did not change with ISIs.

Figure 3 shows the ECD locations in Subject 2 with respect to various check sizes (left panel) and ISIs (right panel). Across subjects, we did not observe a systematic effect of check size or ISI on P100m localization. P100m ECDs to different checks or ISIs were distributed in a relatively confined region around or near the calcarine sulcus.

\section{DISCUSSION}

In the present study the left-half field patterns, with $2^{\circ}$ central occlusion, stimulated mainly parafoveal and peripheral retinal cells. We found that the optimum check size for tuning $\mathrm{P} 100 \mathrm{~m}$ activation was $120^{\prime}$, clearly larger than the $30^{\prime}$ checks in earlier VEP recordings. Taking the retinotopic organization into

Table 2: Mean amplitudes (nAm), peak latencies (ms), and locations (x-, y-, z- coordinates; mm) of P100m sources to checkerboard stimulation in ten subjects with respect to various ISI

\begin{tabular}{lrrrrrrr}
\hline ISI (s) & $\mathbf{0 . 1 6}$ & $\mathbf{0 . 1 8}$ & $\mathbf{0 . 2 5}$ & $\mathbf{0 . 5}$ & $\mathbf{1}$ & $\mathbf{2}$ & $\mathbf{4}$ \\
Amplitude & 43.1 & 46.0 & 32.1 & 34.1 & 36.8 & 34.5 & 34.0 \\
SD & 18.0 & 16.6 & 7.0 & 11.0 & 14.0 & 11.8 & 12.3 \\
Latency & 99.0 & 100.7 & 103.0 & 103.3 & 103.5 & 99.4 & 98.3 \\
SD & 6.3 & 7.5 & 4.5 & 6.1 & 6.0 & 4.0 & 4.2 \\
X value & 14.6 & 14.1 & 17.1 & 16.2 & 16.6 & 19.4 & 17.9 \\
SD & 5.1 & 5.3 & 4.8 & 7.1 & 6.7 & 5.4 & 5.1 \\
y value & -39.8 & -40.9 & -41.4 & -40.8 & -39.0 & -38.8 & -39.0 \\
SD & 8.2 & 7.4 & 7.1 & 7.5 & 7.0 & 7.7 & 6.5 \\
Z value & 42.8 & 44.1 & 45.1 & 43.0 & 44.8 & 43.9 & 43.8 \\
SD & 4.7 & 5.2 & 3.8 & 5.4 & 8.7 & 4.8 & 3.0
\end{tabular}




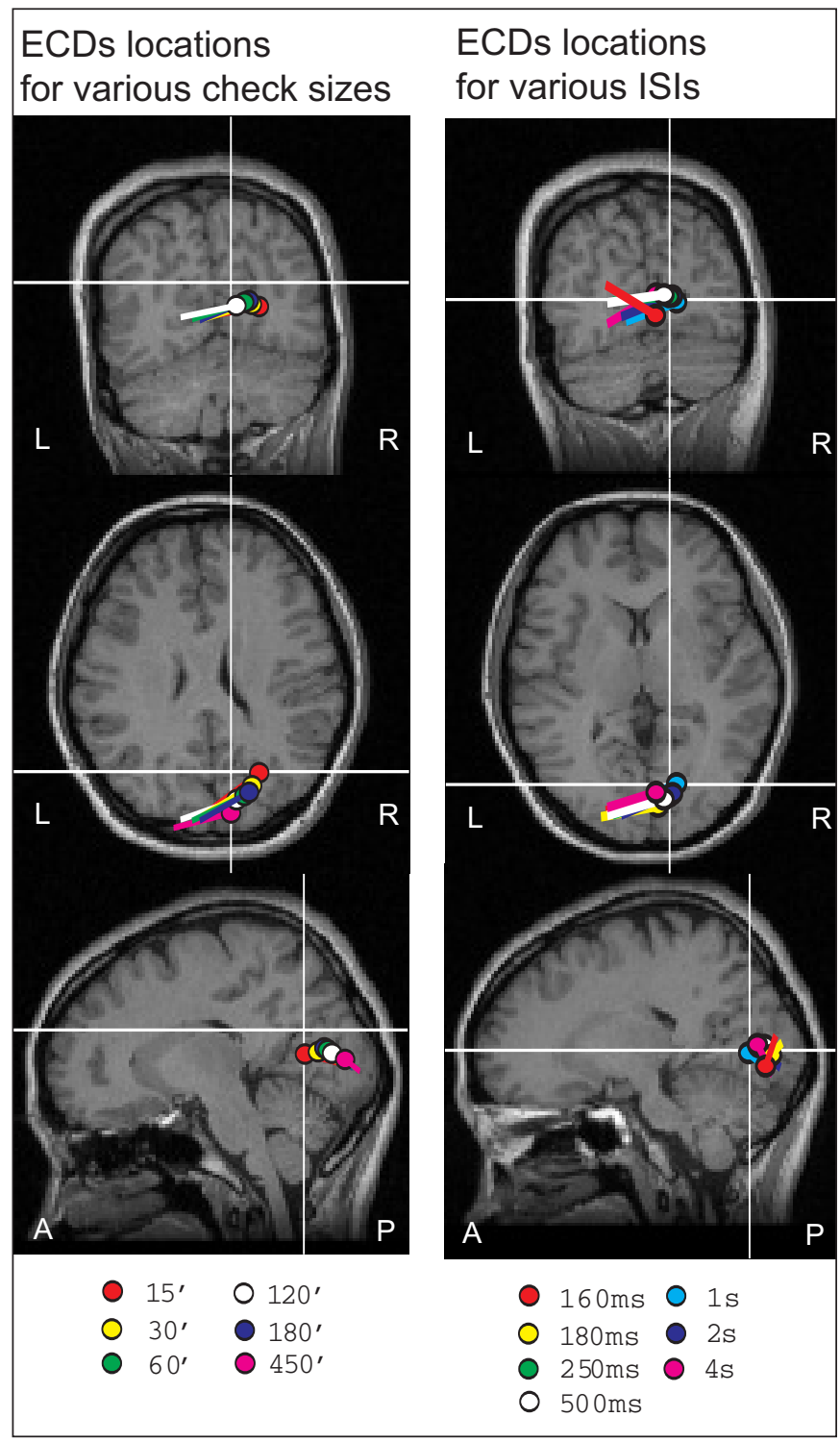

Figure 3: The MRI-overlaid equivalent current dipole (ECD) locations with respect to various check sizes (left column) and interstimulus intervals (ISIs, right column). Only responses to six different check sizes or ISI values are shown for simplicity. Note the generators of P100m responses are localized near or around the calcarine sulcus.

consideration, large checks (low spatial frequency) preferentially activate peripheral retinal cells and anterior striate cortex, hence powerful for P100m elicitation. ${ }^{11,12}$ In contrast, small-element patterns (high spatial frequency) preferentially activate fovea retinal cells and posterior striate cortex (occipital pole), and thus are relatively suitable for eliciting P100 rather than $\mathrm{P} 100 \mathrm{~m}$.

The VEF response to checkerboard pattern-reversal stimulation in our study is basically a triphasic wave complex with a large second deflection peaking at $\sim 100 \mathrm{~ms}$, compatible with the N75m-P100m-N145m complex demonstrated by earlier VEF studies. ${ }^{11,13,14}$ Despite lack of simultaneous EEG recording, we putatively assume that P100m corresponds to P100 VEP response since it was consistently associated with one earlier $\sim 70$ $\mathrm{ms}$ and one later $\sim 150 \mathrm{~ms}$ opposite deflection. Previous VEF studies using simultaneous EEG recording did not demonstrate significant latency differences between P100m and P100, suggesting similar cortical activation for both. ${ }^{11,13,14}$ Notably, our mean latency of P100m to $30^{\prime}$ checks (115.0 ms, see Table 1) approximates that $(118.8 \mathrm{~ms})$ to $28^{\prime}$ checks in one previous VEF study ${ }^{14}$ using similar recording conditions.

In our study, the P100m responses to small check size (15') were characteristic of relatively small amplitudes and delayed peak latencies, in line with earlier $\operatorname{VEP}^{3,15}$ and one recent $\mathrm{MEG}^{13}$ study. Based on the assumption of parallel visual processing, 3,16 retinal ganglion cells most sensitive to the high spatial frequency stimuli are mainly localized in fovea areas and are characterized by the relatively small receptive fields and small diameter axons. This assumption supported our observation that small checks were less powerful for $\mathrm{P} 100 \mathrm{~m}$ elicitation by peripheral stimulation. The preferential activation of ganglion cells with small axon and slow conduction may contribute to the latency prolongation.

The effect of checks $>120^{\prime}$ on VEFs was rarely discussed. Nakamura and colleagues ${ }^{13}$ reported a slightly longer latency and smaller amplitude of P100m by $180^{\prime}$ than $90^{\prime}$ checks. ${ }^{13}$ In our study, P100m activation to $180^{\prime}$ and $450^{\prime}$ checks showed consecutive attenuation compared with that to $120^{\prime}$ checks. We had two explanations for this finding. First, the sensitivity to pattern stimuli decreased when small numbers of pattern cycle was displayed. ${ }^{17}$ Decrease in P100m activation to $180^{\prime}$ and $450 '$ checks probably reflected the scarce number of pattern cycles presented (five cycles for 180', two cycles for 450') in this study. Notably, the optimum 120' checks stimulation encompassed around six pattern cycles. This number was intriguingly in line with a color contrast study ${ }^{18}$ which demonstrated attenuated visual evoked responses when less than six cycles of isoluminant red/green sinusoid gratings were displayed. An alternative explanation for the P100m attenuation by $180^{\prime}$ and 450 ' checks is that the VEF responses to very large checks may not exclusively reflect contrast processing. The preferential activation of luminance channels at large checks might confound the contrastspecific responses, hence an attenuated VEF magnitude. An earlier study on pattern-reversal retinal potentials has shown that the retinal activation by large checks could be confounded by noncontrast specific responses. ${ }^{19}$

Apart from P100 or P100m, check size also affects N75 responses. Several VEP studies showed decreased amplitude and latency of N75 when the check size increases. ${ }^{7,20,21}$ Moreover, $\mathrm{N} 75$ or $\mathrm{N} 75 \mathrm{~m}$, like $\mathrm{P} 100$ or $\mathrm{P} 100 \mathrm{~m}$, is also affected by aging ${ }^{22}$ and by contrast level. ${ }^{14,23}$ Taken together, further studies investigating the check size effect upon N75m in VEF response should be intriguing and may help elucidate the neurophysiological significance of $\mathrm{N} 75 \mathrm{~m}$. In this paper, however, we did not include this issue, because the stimulus paradigm with a wide check size variation did not consistently elicit discernible N75m responses (see Figure 1).

The ISI effect on $\mathrm{P} 100 \mathrm{~m}$ has been rarely studied. One previous VEP study reported a latency shortening of $4.8 \mathrm{~ms}$ as the ISI increased from $0.25 \mathrm{~s}$ to $1 \mathrm{~s}^{24}$ In contrast, our results showed no significant ISI-dependent change in $\mathrm{P} 100 \mathrm{~m}$ activation. Note the ISI less than $0.16 \mathrm{~s}$ was not introduced in the present study since the transient VEP or VEF responses (P100, 
P100m) would be blurred, producing steady-state responses when the reversal rate becomes higher than $3.5 \mathrm{~Hz}$ (ISI $\leq 0.14$ s). ${ }^{25}$ The evoked cortical responses typically first increase monotonously with the increment of ISI and then reach a saturation level. ${ }^{26}$ The reduced strengths at short ISI may be related to neuronal refractoriness. Our observation indicates a very short refractory period $(<0.16 \mathrm{~s})$ for primary visual cortex activation. The clear activation by short ISIs may be supported by the preferential responses of $\mathrm{M}$ retinal ganglion cells to stimuli with high temporal frequency and low spatial frequency. ${ }^{4}$

One alternative explanation is the additional activation from dorsal stream which resulted from the movement sensations inevitably occurring in pattern reversals, especially when ISI is short. ${ }^{27}$ This motion-like response and the averaging property of both the onset- and offset- response in pattern-reversal VEPs or VEFs probably explain why few researchers focused on the ISI effect using this stimulation mode. Despite the above, to clarify the systemic ISI effect in the present setting is important, since pattern-reversing remains a major stimulation mode in clinical practices. Further application of the pattern on/off stimulation, which has strength in avoidance of movement sensation and habituation $^{27}$ might help elucidate the visual neuromagnetic responses to ISI variation.

Contrary to the controversial localization of P100 generators in striate or extrastriate origins in VEP studies, ${ }^{28,29}$ earlier ${ }^{10,13}$ and our present MEG studies showed unitary localization of $\mathrm{P} 100 \mathrm{~m}$ around or near the calcarine fissure. Based on Novak's hypothesis, ${ }^{3}$ visual stimulations with different spatial frequencies might elicit corresponding neuronal responses. However, using a whole-scalp MEG, we did not demonstrate a systematic change of source locations with check size variation. P100m sources did not differ significantly in location with ISI, either. We thus inferred that the representation areas for different spatial and ISI conditions might be considerably overlapping. Of note, applying single dipole model for source localization inevitably summated all the activated areas as "a center of gravity", even if these activations are isolated in localizations. Therefore, the localization around the calcarine fissure in this study might reflect the summated activation of the upper and lower parts of the anterior striate. However, the use of single dipole model, in comparison with time-varying multidipole model, is more straightforward and readily satisfies our study's purpose to elucidate the systemic effects of check size variation upon the visual cortical activation.

Although foveal stimulation is important for VEP recordings, MEG activity has a peripheral rather than central field prevalence. ${ }^{11}$ Differential attention effects ${ }^{30}$ and source distributions ${ }^{11}$ have been reported between VEFs to peripheral and central field stimulations. Moreover, the different peripheral and foveal deficits can be observed in some pathological conditions. ${ }^{2,31}$ Thus, understanding of check size and ISI effects on neuromagnetic responses to peripheral field stimulation is important for further MEG studies on the visual cortical functions.

Check size has clear impacts upon the amplitudes and latencies of $\mathrm{P} 100 \mathrm{~m}$ responses, thus one should consider these parameters in studying neuromagnetic visual cortical responses to central-occluded pattern-reversal stimulation. The 120' checks offer the optimal spatial stimuli for eliciting maximum $\mathrm{P} 100 \mathrm{~m}$ activation. With ISIs between 0.16 and $4 \mathrm{~s}$, one can obtain similar $\mathrm{P} 100 \mathrm{~m}$ responses to pattern stimuli in the centraloccluded visual field. Moreover, we found no systematic change in ECD location with reversal rate or check size.

\section{ACKNOWLEDGMENTS}

We thank Mr. Chih-Che Chou and Mr. Chou-Ming Cheng for technical assistance in the acquisition of MRIs, Ms. Wen-Yung Sheng for her invaluable assistance in our statistical analysis. This study was supported by research grants from Taipei Veterans General Hospital (VGH-93, VGH-94-323, Y.Y. LIN) and National Science Council (NSC93-2341-B-075-086, Y.Y. LIN), Taipei, Taiwan.

\section{REFERENCES}

1. Török B, Meyer M, Wildberger $\mathrm{H}$. The influence of pattern size on amplitude, latency and wave form of retinal and cortical potentials elicited by checkerboard pattern reversal and stimulus onset-offset. Electroenceph Clin Neurophysiol 1992; 84: 13-19.

2. Plant GT, Hess RF. Temporal frequency discrimination in optic neuritis and multiple sclerosis. Brain 1985; 108: 647-676.

3. Novak GP, Wiznitzer M, Kurtzberg D, Giesser BS, Vaughan HG Jr. The utility of visual evoked potentials using hemifield stimulation and several check sizes in the evaluation of suspected multiple sclerosis. Electroenceph Clin Neurophysiol 1988; 71: 1-9.

4. Merigan WH, Maunsell JHR. How parallel are the primate visual pathways? Ann Rev Neurosci 1993; 16: 369-402.

5. Oelkers R, Grosser K, Lang E, et al. Visual evoked potentials in migraine patients: alterations depend on pattern spatial frequency. Brain 1999; 122: 1147-1155.

6. Kergoat H, Kergoat M-J, Justino L, et al. Visual retinocortical function in dementia of the Alzheimer type. Gerontology 2002; 48: 197-203.

7. Kurita-Tashima S, Tobimatsu S, Nakayama-Hiromatsu M, Kato M. Effect of check size on the pattern reversal visual evoked potential. Electroenceph Clin Neurophysiol 1991; 80: 161-166.

8. Hämäläinen M, Hari R, Ilmoniemi RJ, Knuutila J, Lounasmaa OV. Magnetoencephalography-theory, instrumentation, and applications to noninvasive studies of the working human brain. Rev Mod Phys 1993; 65: 413-497.

9. Barrett G, Blumhardt L, Halliday AM, Halliday E, Kriss A. A paradox in the lateralisation of the visual evoked response. Nature 1976; 261: 253-255.

10. Nakamura A, Kakigi R, Hoshiyama M, et al. Visual evoked cortical magnetic fields to pattern reversal stimulation. Cogn Brain Res 1997; 6: 9-22.

11. Brecelj J, Kakigi R, Koyama S, Hoshiyama M. Visual evoked magnetic responses to central and peripheral stimulation: simultaneous VEP recordings. Brain Topogr 1998; 10: 227-237.

12. Ahlfors SP, Ilmoniemi RJ, Hämäläinen MS. Estimates of visually evoked cortical currents. Electroenceph Clin Neurophysiol 1992; 82: 225-236.

13. Nakamura M, Kakigi R, Okusa T, Hoshiyama M, Watanabe K. Effects of check size on pattern reversal visual evoked magnetic field and potential. Brain Res 2000; 872: 77-86.

14. Hashimoto T, Kashii S, Kikuchi M, et al. Temporal profile of visual evoked responses to pattern-reversal stimulation analyzed with a whole-head magnetometer. Exp Brain Res 1999; 125: 375-382.

15. Chiappa KH, Gill EM, Lentz KE. Effect of check size on P100 latency. Electroenceph Clin Neurophysiol 1985; 61: 29P-30P.

16. Silveira LC, Perry VH. The topography of magnocellular projecting ganglion cells (M-ganglion cells) in the primate retina. Neuroscience 1991; 40: 217-237.

17. Anderson SJ, Burr DC. Receptive field size of human motion detection units. Vision Res 1987; 27: 621-635.

18. Fylan F, Holliday IE, Singh KD, Anderson SJ, Harding GFA. Magnetoencephalographic investigation of human cortical area V1 using color stimuli. Neuroimage 1997; 6: 47-57.

19. Trick GL, Wintermeyer DH. Spatial and temporal frequency tuning 
of pattern-reversal retinal potentials. Invest Ophthalmol Vis Sci 1982; 23: 774-779.

20. Bodis-Wollner I, Brannan JR, Nicoll J, Frkovic S, Mylin LH. A short latency cortical component of the foveal VEP is revealed by hemifield stimulation. Electroenceph Clin Neurophysiol 1992; 84: 201-208.

21. Onofrj M, Fulgente T, Thomas A, et al. Visual evoked potentials generator model derived from different spatial frequency stimuli of visual field regions and magnetic resonance imaging coordinates of V1, V2, V3 areas in man. Int J Neurosci 1995; 83: 213-239.

22. Allison T, Hume AL, Wood CC, Goff WR. Developmental and aging changes in somatosensory, auditory and visual evoked potentials. Electroenceph Clin Neurophysiol 1984; 58: 14-24

23. Previc FH. The neurophysiological significance of the N1 and P1 components of the visual evoked potential. Clin Vision Sci 1988; 3: $195-202$.

24. Stockard JJ, Hughes JF, Sharbrough FW. Visually evoked potentials to electronic pattern reversal: latency variations with gender, age, and technical factors. Am J EEG Technol 1979; 19: 171-204.

25. Celesia GG, Bodis-Wollner I, Chatrian GE, et al. Recommended standards for electroretinograms and visual evoked potentials.
Report of an IFCN Committee. Electroenceph Clin Neurophysiol 1993; 87: 421-436.

26. Uusitalo MA, Jousmäki V, Hari R. Activation trace lifetime of human cortical responses evoked by apparent visual motion. Neurosci Lett 1997; 224: 45-48.

27. Tzelepi A, Ioannides AA, Poghosyan V. Early (N70m) neuromagnetic signal topography and striate and extrastriate generators following pattern onset quadrant stimulation. Neuroimage 2001; 13: 702-718.

28. Strucl M, Prevec TS, Zidar I. Dependence of visual evoked potentials on change of stimulated retinal area associated with different pattern displacements. Electroenceph Clin Neurophysiol 1982; 53: 634-642.

29. Maier J, Dagnelie G, Spekreijse H, van Dijk BW. Principal components analysis for source localization of VEPs in man. Vision Res 1987; 27: 165-177.

30. Hoshiyama M, Kakigi R. Effects of attention on pattern-reversal visual evoked potentials: foveal field stimulation versus peripheral field stimulation. Brain Topogr 2001; 13: 293-298.

31. Vleugels L, Charlier M, van Nunen A, et al. Temporal resolution deficits in the visual fields of MS patients. Vision Res 1999; 39: 2429-2438. 